\title{
Inducible Nitric Oxide Synthase Genetic Polymorphism and Risk of Asbestosis
}

\author{
Alenka Franko, ${ }^{1}$ Metoda Dodič-Fikfak, ${ }^{1}$ Niko Arnerić, ${ }^{1}$ and Vita Dolžan ${ }^{2}$ \\ ${ }^{1}$ Clinical Institute of Occupational Medicine, University Medical Center, Poljanski Nasip 58, 1000 Ljubljana, Slovenia \\ ${ }^{2}$ Institute of Biochemistry, Faculty of Medicine, University of Ljubljana, Vrazov trg 2, 1000 Ljubljana, Slovenia
}

Correspondence should be addressed to Vita Dolžan, vita.dolzan@mf.uni-lj.si

Received 27 September 2010; Revised 10 March 2011; Accepted 23 March 2011

Academic Editor: Celina Janion

Copyright (C) 2011 Alenka Franko et al. This is an open access article distributed under the Creative Commons Attribution License, which permits unrestricted use, distribution, and reproduction in any medium, provided the original work is properly cited.

\begin{abstract}
Asbestos, a known occupational pollutant, may upregulate the activity of inducible nitric oxide synthase (iNOS) and thus the production of nitric oxide (NO). This study investigated whether $i N O S$ (CCTTT) nolymorphism is associated with an increased asbestosis risk in exposed workers. The study cohort consisted of 262 cases with asbestosis and 265 controls with no asbestos-related disease. For each subject the cumulative asbestos exposure data were available. The number of CCTTT repeats was determined following PCR amplification of the iNOS promoter region. Logistic regression was performed to estimate asbestosis risk. The OR of asbestosis was 1.20 (95\% CI $=0.85-1.69)$ for the LL genotype compared to the combined SL and SS genotypes and 1.26 (95\% $\mathrm{CI}=0.86-1.85)$ for the LL genotype compared to the SL genotype. The results of this study are borderline significant and suggest a possible role of $i$ NOS (CCTTT) $)_{n}$ polymorphism in the risk of asbestosis; however, further studies are needed.
\end{abstract}

\section{Introduction}

Asbestosis is one of the most frequently studied diseases associated with the inhalation of asbestos fibers. The exposure to asbestos is known to cause inflammation, fibrous scarring in the lung, and cancer $[1,2]$. It has been suggested that, in addition to reactive oxygen species (ROS), the reactive nitric species (RNS), such as nitric oxide (NO), may also be involved in the development of asbestos-related diseases [36].

Nitric oxide is a free radical which readily reacts with ROS, such as superoxide anion, producing peroxynitrite, which can generate hydroxyl radicals [7-11]. In the living organisms NO is synthesized by nitric oxide synthases (NOSs), which catalyse the conversion of amino acid L-arginine and molecular oxygen into NO and L-citrulline [11, 12]. Three isoforms of NOS have been identified: neuronal NOS (nNOS or NOS1), inducible NOS (iNOS or NOS2), and endothelial NOS (eNOS or NOS3) [7]. Inducible NOS is often present at sites of chronic inflammation $[5,11]$.

Nitric oxide generation is thought to be an important mediator of cell injury and inflammation caused by asbestos [13]. Studies indicate that asbestos fibers may upregulate the activity of iNOS and thus the production of NO by alveolar macrophages and pulmonary epithelial cells $[5,6$, 13-15]. Several studies described the association between NO production and the adverse effects of asbestos $[3-6,13]$. Chao et al. reported an association between the magnitude of NO production by lung epithelial cells and DNA oxidation at in vitro exposure to asbestos [3]. Treatment of human lung epithelial cells with asbestos was shown to result in the synthesis of iNOS mRNA and increased intracellular levels of nitrate, a stable oxidation product of NO [3]. In addition, a temporal correlation was demonstrated between NO production by alveolar macrophages and neutrophil infiltration into lung after inhalation of asbestos [13]. The findings of these studies suggest that $\mathrm{NO}$ may play an important role in the initiation and progression of asbestosis [6]. However, the studies in iNOS knockout mice indicate that iNOS-derived NO exerts a dual role in acute asbestosinduced lung injury as iNOS deficiency resulted in an exacerbated inflammatory response but improved oxidantpromoted lung tissue damage [16].

The human iNOS gene is polymorphic. Several types of polymorphisms have been identified in the promoter region of the iNOS gene, among them the tandem repeat number 
polymorphism of a CCTTT pentanucleotide $\left((\text { CCTTT })_{\mathrm{n}}\right)$ $[17,18]$. The CCTTT pentanucleotide repeats within the promotor region of the human iNOS gene have been associated with the biochemical promoter activity [19]. Several studies have suggested an association between inflammatory diseases or cancer and the number of the CCTTT repeats [18-21]. However, to our knowledge and according to the literature available, this polymorphism has not been studied so far in association with asbestosis.

In this study we investigated whether iNOS (CCTTT) ${ }_{n}$ polymorphism is associated with an increased risk of asbestosis in workers exposed to asbestos.

\section{Methods}

The study population comprised 262 subjects who were diagnosed with asbestosis as an occupational disease and 265 subjects with no asbestos disease as the control group. Cases and controls were matched by gender and age. All the subjects were selected from a cohort of 2080 workers who were occupationally exposed to asbestos in the asbestos cement manufacturing plant of Salonit Anhovo, Slovenia, and presented at the State Board for Recognition of Occupational Asbestos Diseases in the period between 1 January 1998 and 31 December 2003 [22].

To obtain the information about smoking, all cases and controls were interviewed by the same investigator, using a standardized questionnaire $[23,24]$. For each subject included in the present study, the cumulative asbestos exposure data were obtained from the previous study [24].

The diagnosis of asbestosis or "no asbestos-related disease" was confirmed by two groups of experienced occupational experts of the State Board for Recognition of Occupational Asbestos Diseases at the Clinical Institute of Occupational Medicine. Each group of experts consisted of an occupational physician, a radiologist, and a pulmonologist skilled in diagnosis of asbestos-related diseases. The diagnosis was based on the Helsinki Criteria for Diagnosis and Attribution of Asbestos Diseases [25] and on the American Thoracic Society recommendations [26]. HighResolution Computer Tomography (HRCT) was used for radiological diagnosis of asbestosis. In about $1 \%$ of subjects, the histopathological examination was performed as well.

Genotyping of the (CCTTT $)_{n}$ in the iNOS gene promoter was performed by the amplification of the corresponding region of the promoter by PCR using a primer pair with one primer being fluorescently labelled [27]. The amplified fragments were analysed on the ABI PRISM 310 Genetic Analyser (Applied Biosystems), and the number of polymorphic repeats was determined with GeneMapper analysis software (Applied Biosystems, Norwalk, USA) as previously reported [28].

First, the statistical analysis included the descriptive statistics, $t$-test, and $\chi^{2}$ test. Next, univariate logistic regression was performed to estimate the risk of asbestosis in relation to individual variables (iNOS, cumulative asbestos exposure, gender, age, and smoking). These analyses were followed by multivariate logistic regression modelling.
TABLE 1: Allele frequencies of the iNOS (CCTTT) $)_{n}$ gene polymorphism in cases and controls.

\begin{tabular}{lccc}
\hline No. of CCTTT repeats & $\begin{array}{c}\text { Size } \\
(\mathrm{bp})\end{array}$ & $\begin{array}{c}\text { Cases } \\
N(\%)\end{array}$ & $\begin{array}{c}\text { Controls } \\
N(\%)\end{array}$ \\
\hline 6 & 166 & 1 & 1 \\
7 & 171 & 1 & 2 \\
8 & 176 & 0 & 2 \\
9 & 181 & 20 & 22 \\
10 & 186 & 72 & 71 \\
11 & 191 & 75 & 82 \\
12 & 196 & 193 & 173 \\
13 & 201 & 93 & 96 \\
14 & 206 & 32 & 33 \\
15 & 211 & 24 & 31 \\
16 & 216 & 3 & 3 \\
17 & 221 & 2 & 1 \\
18 & 226 & 0 & 1 \\
\hline$\leq 11$ (short alleles) & & 169 & 180 \\
$\geq 12$ (long alleles) & & 347 & 338 \\
\hline
\end{tabular}

\section{Results}

The subjects with asbestosis were on average 61 and the controls 57 years old $(t=5.18, P=.000)$. Comparison of smoking between the cases and the controls showed no statistically significant difference $\left(\chi^{2}=0.01, P=.919\right)$. Approximately forty-five percent of subjects (117 cases and 120 controls) were ever smokers.

The average cumulative asbestos exposure was significantly higher in subjects with asbestosis compared to those without asbestos-related disease $\left(37.67 \mathrm{fibres} / \mathrm{cm}^{3}\right.$-years for cases and 11.23 fibres $/ \mathrm{cm}^{3}$-years for controls; $=4.78, P=$ $.000)$.

Genotyping of the (CCTTT $)_{n}$ in the iNOS gene promoter was successful in 258 cases and 259 controls. Thirteen alleles for the iNOS microsatellite ranging from 166 to 226 base pears (bp) (6 to 18 CCTTT repeats) were observed (Table 1 ). The alleles with $\leq 11$ CCTTT repeats were defined as short alleles (S) and alleles with $\geq 12$ repeats as long alleles (L) (Table 1).

Accordingly, the subjects were classified into three groups of genotypes: SS, SL, and LL. Inducible nitric oxide synthase LL genotype was observed in 132 cases and 121 controls, SL genotype in 83 cases and 96 controls, while SS genotype was found in 43 cases and 42 controls (Table 2). When combining genotypes, no significant difference was found in the frequency of the iNOS LL genotype versus the frequency of combined SL and SS genotypes (Table 2).

No association has been observed between asbestosis and smoking (ever/never) $(\mathrm{OR}=0.98,95 \% \mathrm{CI}=0.69$ 1.39). Cumulative asbestos exposure was logarithmically transformed, leading to an OR of asbestosis of 3.21 (95\% $\mathrm{CI}=2.43-4.23)$. 
TABLE 2: Frequencies of iNOS genotypes in cases and controls.

\begin{tabular}{lcccc}
\hline & \multicolumn{2}{c}{ Cases $(n=258)$} & \multicolumn{2}{c}{ Controls $(n=259)$} \\
iNOS genotype & $N$ & $\%$ & $n$ & $\%$ \\
\hline LL & 132 & 51.2 & 121 & 46.7 \\
SL & 83 & 32.1 & 96 & 37.1 \\
SS & 43 & 16.7 & 42 & 16.2 \\
SL + SS & 126 & 48.8 & 138 & 53.3 \\
\hline
\end{tabular}

TABLE 3: Risk of asbestosis for the iNOS LL genotype versus the combined SL and SS genotypes after adjustment by gender, age, smoking, and cumulative asbestos exposure.

\begin{tabular}{lcc}
\hline & OR & $95 \%$ CI \\
\hline Unadjusted & 1.20 & $0.85-1.69$ \\
Adjusted by & & \\
$\quad$ Gender & 1.20 & $0.85-1.70$ \\
Age & 1.19 & $0.84-1.69$ \\
Smoking (ever/never) & 1.17 & $0.83-1.66$ \\
$\quad$ Cumulative exposure & 1.19 & $0.82-1.73$ \\
\hline
\end{tabular}

Determining the associations between $i N O S$ genotypes and asbestosis, the OR of asbestosis was 1.07 (95\% CI $=0.65$ 1.74 ) for the LL genotype versus SS genotype, 1.26 (95\% CI $=0.86-1.85)$ for the LL genotype compared to SL genotype, $0.84(95 \%$ CI $=0.50-1.42)$ for the SL genotype versus SS genotype, and 1.20 (95\% CI = 0.85-1.69) for the LL genotype compared to the combined SL and SS genotypes. The risk of asbestosis for the iNOS LL genotype versus the combined SL and SS genotypes remained practically unchanged after adjustment by gender, age, smoking, and cumulative asbestos exposure (Table 3 ).

\section{Discussion}

It has been suggested that iNOS-derived NO may play an important role in the development of asbestos-related diseases $[5,6,13-15]$. In a nested case-control study the authors investigated the influence of the iNOS (CCTTT) polymorphism on the risk of developing asbestosis. A slightly (20\%) higher risk of asbestosis for the iNOS LL genotype compared to the combined SL and SS genotypes and almost $30 \%$ for the LL versus SL genotype has been detected. This finding, although borderline significant, could be in agreement with the observations of some other studies which demonstrated an association between a disease and longer forms of $(\text { CCTTT })_{\mathrm{n}}$ repeat in the iNOS promoter region $[18,20,21]$. These results could be explained by a higher promoter activity of the $i N O S$ gene which has been shown to increase with the (CCTTT) $)_{n}$ repeat number in an in vitro study [19] and consequently increased production of NO. Since NO is a free radical and its production has been associated with pulmonary damage, inflammation, and disease progression caused by asbestos $[5,6]$, our finding could be considered as biologically plausible.

All the cases and controls who participated in this study were recruited in a small geographical area with an ethnically homogenous population [29]. Therefore, no bias was introduced by genetic heterogeneity.

Although the Board of experts used the HRCT as a widely recommended method for the diagnosis of asbestosis $[25,26]$ in all subjects, there is still a slight possibility that some subjects might have been recruited as controls when they had already developed asbestosis, which could be diagnosed only by the histopathological examination. In that case, we would have more subjects with asbestosis. Considering that our study included cases who had iNOS LL genotype more frequently than the controls, the association between asbestosis and iNOS LL genotype might have been slightly stronger in that case.

In conclusion, to our knowledge, this is the first study investigating the association between asbestosis and iNOS genetic polymorphism. Considering that the results of the current study are borderline significant, further studies including a larger number of subjects are needed to clarify this association.

\section{Acknowledgment}

This work was financially supported by the Slovenian Research Agency (ARRS Grants no. P1-0170-0381 and no. L3-9129-0381).

\section{References}

[1] M. R. Becklake, "Exposure to asbestos and human disease," New England Journal of Medicine, vol. 306, no. 24, pp. 14801482, 1982.

[2] J. E. Craighead and B. T. Mossman, "The pathogenesis of asbestos-associated diseases," New England Journal of Medicine, vol. 306, no. 24, pp. 1446-1455, 1982.

[3] C. C. Chao, S. H. Park, and A. E. Aust, "Participation of nitric oxide and iron in the oxidation of DNA in asbestostreated human lung epithelial cells," Archives of Biochemistry and Biophysics, vol. 326, no. 1, pp. 152-157, 1996.

[4] S. H. Park and A. E. Aust, "Participation of iron and nitric oxide in the mutagenicity of asbestos in hgprt, gpt+ Chinese hamster V79 cells," Cancer Research, vol. 58, no. 6, pp. 11441148, 1998.

[5] E. Aldieri, D. Ghigo, M. Tomatis et al., "Iron inhibits the nitric oxide synthesis elicited by asbestos in murine macrophages," Free Radical Biology and Medicine, vol. 31, no. 3, pp. 412-417, 2001.

[6] V. Castranova, "Role of nitric oxide in the progression of pneumoconiosis," Biochemistry, vol. 69, no. 1, pp. 32-37, 2004.

[7] U. Förstermann and H. Kleinert, "Nitric oxide synthase: expression and expressional control of the three isoforms," Naunyn-Schmiedeberg's Archives of Pharmacology, vol. 352, no. 4, pp. 351-364, 1995.

[8] T. DeRojas-Walker, S. Tamir, H. Ji, J. S. Wishnok, and S. R. Tannenbaum, "Nitric oxide induces oxidative damage in addition to deamination in macrophage DNA," Chemical Research in Toxicology, vol. 8, no. 3, pp. 473-477, 1995.

[9] J. S. Beckman and W. H. Koppenol, "Nitric oxide, superoxide, and peroxynitrite: the good, the bad, and the ugly," American Journal of Physiology, vol. 271, no. 5, pp. C1424-C1437, 1996. 
[10] D. A. Wink and J. B. Mitchell, "Chemical biology of nitric oxide: insights into regulatory, cytotoxic, and cytoprotective mechanisms of nitric oxide," Free Radical Biology and Medicine, vol. 25, no. 4-5, pp. 434-456, 1998.

[11] B. Halliwell and J. M. C. Gutteridge, "The chemistry of free radicals and related "reactive species"," in Free Radicals in Biology and Medicine, B. Halliwell and J. M. C. Gutteridge, Eds., pp. 73-82, Oxford University Press, Oxford, UK, 3rd edition, 1999.

[12] P. Vallance and J. Collier, "Biology and clinical relevance of nitric oxide," British Medical Journal, vol. 309, no. 6952, pp. 453-457, 1994.

[13] T. R. Quinlan, K. A. BeruBe, M. P. Hacker et al., "Mechanisms of asbestos-induced nitric oxide production by rat alveolar macrophages in inhalation and in vitro models," Free Radical Biology and Medicine, vol. 24, no. 5, pp. 778-788, 1998.

[14] G. Thomas, T. Ando, K. Verma, and E. Kagan, "Asbestos fibers and interferon-gamma up-regulate nitric oxide production in rat alveolar macrophages," American Journal of Respiratory Cell and Molecular Biology, vol. 11, no. 6, pp. 707-715, 1994.

[15] S. Tanaka, N. Choe, D. R. Hemenway, S. Zhu, S. Matalon, and E. Kagan, "Asbestos inhalation induces reactive nitrogen species and nitrotyrosine formation in the lungs and pleura of the rat," Journal of Clinical Investigation, vol. 102, no. 2, pp. 445-454, 1998.

[16] M. Dörger, A. M. Allmeling, R. Kiefmann, A. Schropp, and F. Krombach, "Dual role of inducible nitric oxide synthase in acute asbestos-induced lung injury," Free Radical Biology and Medicine, vol. 33, no. 4, pp. 491-501, 2002.

[17] W. Xu, L. Liu, P. C. Emson, C. R. Harrington, and I. G. Charles, "Evolution of a homopurine-homopyrimidine pentanucleotide repeat sequence upstream of the human inducible nitric oxide synthase gene," Gene, vol. 204, no. 1-2, pp. 165-170, 1997.

[18] M. Tatemichi, T. Sawa, I. Gilibert, H. Tazawa, T. Katoh, and H. Ohshima, "Increased risk of intestinal type of gastric adenocarcinoma in Japanese women associated with long forms of (CCTTT) N pentanucleotide repeat in the inducible nitric oxide synthase promoter," Cancer Letters, vol. 217, no. 2, pp. 197-202, 2005.

[19] K. M. Warpeha, W. Xu, L. Liu et al., "Genotyping and functional analysis of a polymorphic (CCTTT)n repeat of NOS2A in diabetic retinopathy," FASEB Journal, vol. 13, no. 13, pp. 1825-1832, 1999.

[20] M. C. Martín, A. Martinez, J. L. Mendoza et al., "Influence of the inducible nitric oxide synthase gene (NOS2A) on inflammatory bowel disease susceptibility," Immunogenetics, vol. 59, no. 11, pp. 833-837, 2007.

[21] C. H. Shen, Y. H. Wang, W. C. Wang et al., "Inducible nitric oxide synthase promoter polymorphism, cigarette smoking, and urothelial carcinoma risk," Urology, vol. 69, no. 5, pp. 1001-1006, 2007.

[22] A. Franko, M. Dodič-Fikfak, N. Arnerić, and V. Dolžan, "Manganese and extracellular superoxide dismutase polymorphisms and risk for asbestosis," Journal of Biomedicine and Biotechnology, vol. 2009, Article ID 493083, 2009.

[23] B. G. Ferris, "Epidemiology standardization project (American Thoracic Society)," American Review of Respiratory Disease, vol. 118, no. 6, pp. 1-120, 1978.

[24] M. Dodič Fikfak, D. Kriebel, M. M. Quinn, E. A. Eisen, and D. $\mathrm{H}$. Wegman, "A case control study of lung cancer and exposure to chrysotile and amphibole at a Slovenian asbestos-cement plant," Annals of Occupational Hygiene, vol. 51, no. 3, pp. 261268, 2007.

[25] American Thoracic Society, "Diagnosis and initial management of nonmalignant diseases related to asbestos," American Journal of Respiratory and Critical Care Medicine, vol. 170, no. 6, pp. 691-715, 2004.

[26] D. W. Henderson, J. Rantanen, S. Barnhart et al., "Asbestos, asbestosis, and cancer: the Helsinki criteria for diagnosis and attribution," Scandinavian Journal of Work, Environment and Health, vol. 23, no. 4, pp. 311-316, 1997.

[27] B. Rueda, M. A. López-Nevot, M. Pascual et al., "Polymorphism of the inducible nitric oxide synthase gene in celiac disease," Human Immunology, vol. 63, no. 11, pp. 1062-1065, 2002.

[28] T. Hovnik, V. Dolžan, N. U. Bratina, K. T. Podkrajšek, and T. Battelino, "Genetic polymorphisms in genes encoding antioxidant enzymes are associated with diabetic retinopathy in type 1 diabetes," Diabetes Care, vol. 32, no. 12, pp. 22582262, 2009.

[29] B. Vidan-Jeras, B. Jurca, V. Dolžan, M. Jeras, K. Breskvar, and M. Bohinjec, "Caucasian Slovenian normal," in HLA, D. W. Gjertson and P. I. Terasaki, Eds., pp. 180-181, American Society for Histocompatibility and Immunogenetics, Lenexa, Kan, USA, 1998. 

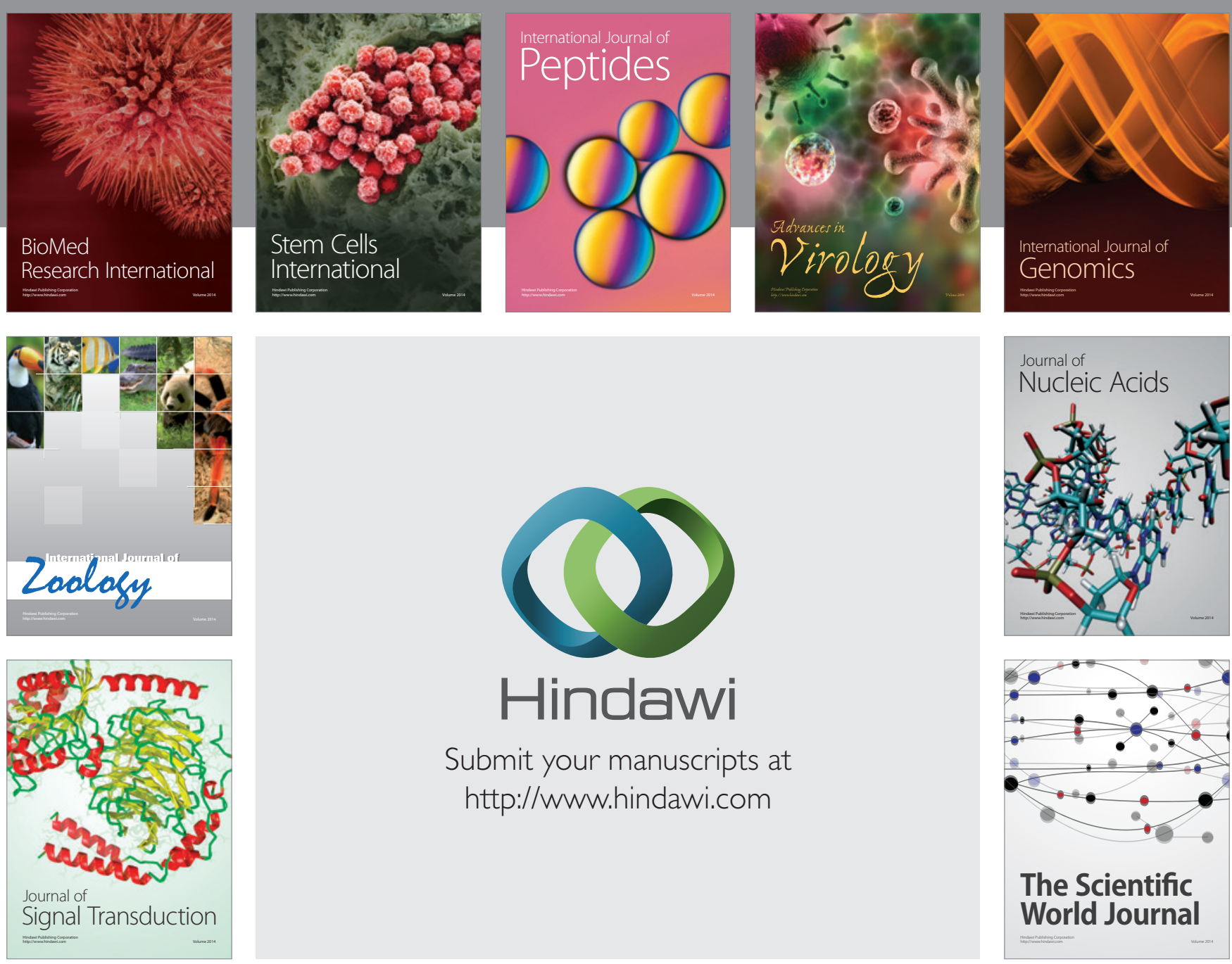

Submit your manuscripts at

http://www.hindawi.com
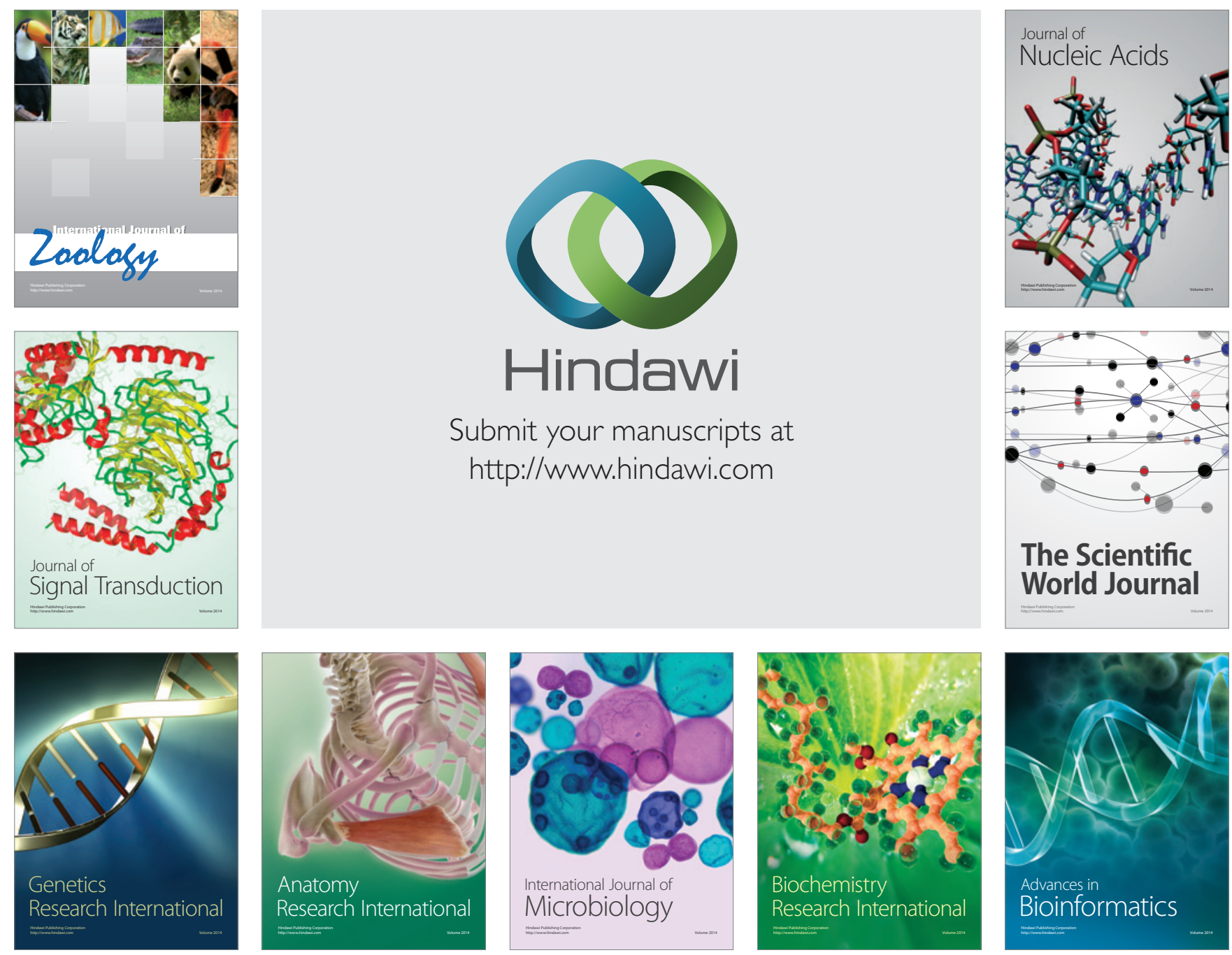

The Scientific World Journal
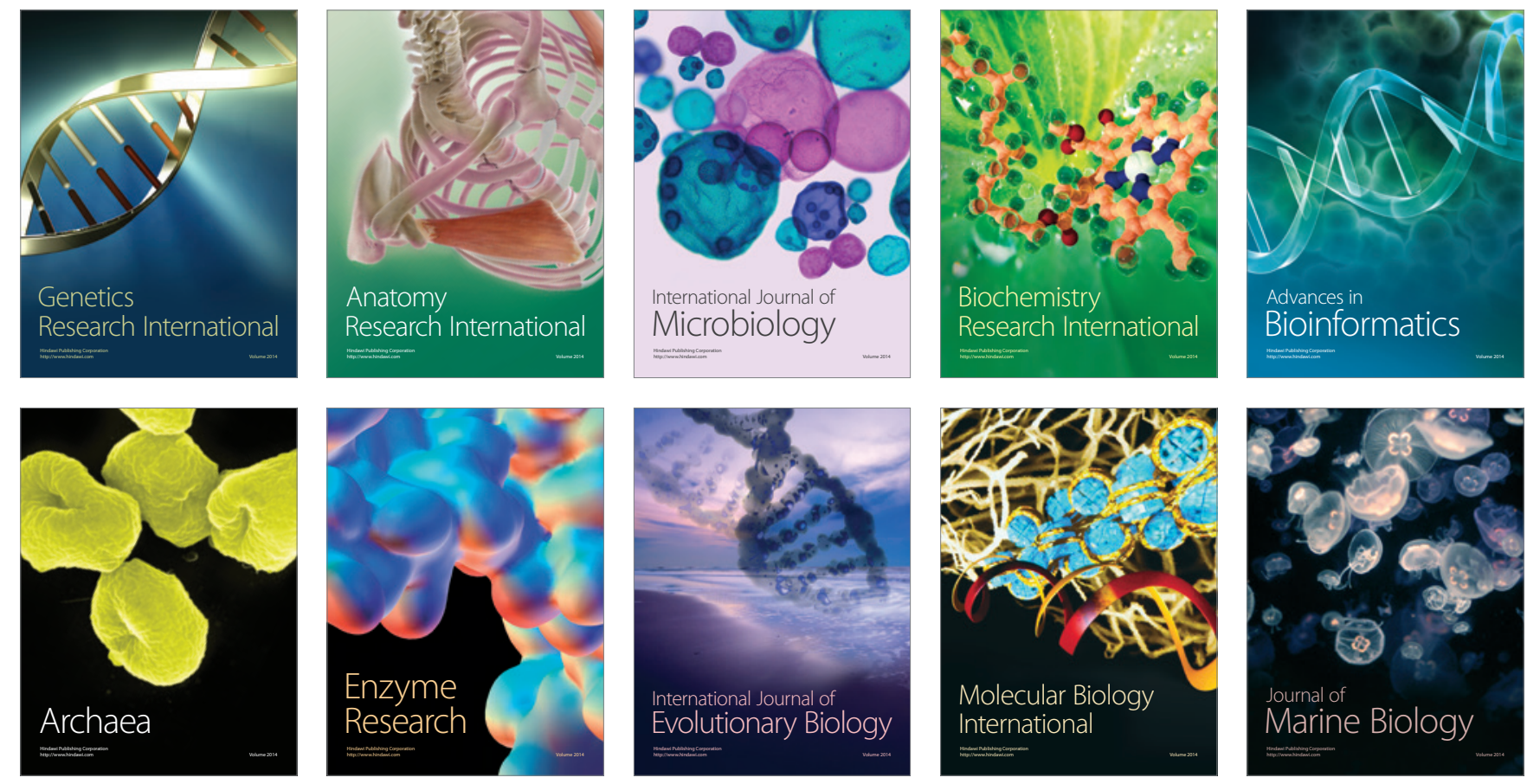